\title{
Long-term results for basilic vein superficialization or transposition for hemodialysis access
}

\author{
Şaban Ergene (iD, Doğuş Hemşinli (D), Sedat Ozan Karakişi (D) \\ Department of Cardiovascular Surgery, Recep Tayyip Erdogan University Medical Faculty, Rize, Turkey
}

\begin{abstract}
Objectives: This study presents our long-term follow-up results of patients undergoing basilic vein superficialization and basilic vein transposition.

Patients and methods: Medical records of a total of 71 patients ( 32 males, 39 females; mean age 56.7 years, range, 31 to 76 years) undergoing brachiobasilic arteriovenous fistula surgery for hemodialysis access between January 2010 and March 2019 were retrospectively analyzed. The patients were divided into two groups according to the type of access procedure as the basilic vein superficialization group ( $\mathrm{n}=42$ ) and basilic vein transposition group ( $\mathrm{n}=29)$. The primary and secondary patency rates were evaluated.

Results: The mean follow-up was 18.8 months. In the early postoperative period, bleeding or hematoma developed in 13 , extremity edema in 11, and superficial wound site infection in four patients. Surgical methods were applied to six of 11 patients who developed fistula thrombosis during follow-up, while interventional methods were applied to five patients. Primary and secondary patency rates were $86 \%$ and $90 \%$, respectively in the superficialization group and $76 \%$ and $90 \%$, respectively in the transposition group. There was no statistically significant difference in the patency rates or postoperative complications between the groups.
\end{abstract}

Conclusion: Owing to their low complication and high patency rates, both basilic vein superficialization and basilic vein transposition can be safely employed in patients in whom arteriovenous fistula cannot be established with the cephalic vein.

Keywords: Arteriovenous fistula, chronic renal insufficiency, hemodialysis.

Long-term hemodialysis support requirements have been growing rapidly due to the increasing number of patients with chronic kidney failure in recent years, longer survival times, and low kidney donor numbers. Therefore, arteriovenous fistula (AVF) creation is required to allow access to hemodialysis for greater numbers of patients. ${ }^{[1]}$ The principal options for arteriovenous access in patients in whom the cephalic vein cannot be employed due to inappropriate structure or previous interventions are brachiobasilic AVF (BBAVF) or arteriovenous graft (AVG). ${ }^{[1-5]}$ Although earlier cannulation is possible with AVG applications, the BBAVF has lower complication and higher patency rates. ${ }^{[3,4]}$ According to the clinical practice guidelines of the European Society for Vascular Surgery published in 2018, autogenous AVF options should be preferred to synthetic grafts for hemodialysis patients owing to their low postoperative complication rates and fewer requirements for endovascular or surgical revision due to AVF failure. Single- or two-stage BBAVF in the upper extremity has been described as a good option, when AVF cannot be established with the cephalic vein, or in the event of failed radial-cephalic or brachial-cephalic AVF. ${ }^{[6]}$

The BBAVF technique was first described by Dagher et al. ${ }^{[7]}$ in 1976. In contrast to other superficial veins in the upper extremity, the basilic vein is

Received: November 13, 2019 Accepted: December 22, 2019 Published online: March 06, 2020

Correspondence: Şaban Ergene, MD. Recep Tayyip Erdoğan Üniversitesi Tıp Fakültesi Kalp ve Damar Cerrahisi Anabilim Dal, 53020 Rize, Türkiye. e-mail: saban.ergene@erdogan.edu.tr 
located deep in the medial arm. Superficialization is required for it to be used for hemodialysis and to avoid damage during repeated cannulation of the median nerve, brachial artery, and medial antebrachial cutaneous nerve accompanying the anatomical course. ${ }^{[1,2]}$ Different surgical techniques have, to date, been described involving single- or two-stage superficialization only or simultaneous transposition of the basilic vein. ${ }^{[1-5,8,9]}$ Several studies have compared the patency and complication rates of BBAVF techniques with those of other AVFs and AVG. ${ }^{[1,2]}$ Promising results have been recorded for BBAVF, and it has entered the guidelines as superior to AVG, in particular. ${ }^{[1,2,6]}$ However, the number of studies comparing patency and complication rates among BBAVF techniques is very limited.

In the present study, we aimed to evaluate the long-term results of patients undergoing basilic vein superficialization (BVS) or basilic vein transposition (BVT) in our clinic.

\section{PATIENTS AND METHODS}

\section{Study design}

Medical records of patients undergoing BBAVF surgery for hemodialysis access due to end stage kidney failure between January 2010 and March 2019 were retrospectively analyzed. Data were collected for patients undergoing BBAVF due to fistulas previously established using the cephalic vein becoming non-functional or to the absence of a suitable cephalic vein. Thirteen patients with missing data were excluded. Finally, a total of 71 patients (32 males, 39 females; mean age 56.7 years, range, 31 to 76 years) constituted the study population. The patients were divided into two groups as BVS and BVT. The surgical technique was selected based on the surgeon's preference. Patients' demographic data, additional diagnoses, operations applied, ultrasonographic data, follow-up times, primary and secondary patency rates, and complication data were recorded and compared for significance between the two groups. The primary patency was defined as successful functioning of the fistula with no intervention following establishment of AVF. Secondary patency was defined as successful fistula functioning irrespective of the number of interventions.

A written informed consent was obtained from each patient. The study protocol was approved by Recep Tayyip Erdoğan University Medical Faculty
Ethics Committee (No. 40986104-799). The study was conducted in accordance with the principles of the Declaration of Helsinki.

\section{Operative technique}

The suitability for AVF creation of the vascular structures in all patients was evaluated using Duplex ultrasonography (USG). Conditions of a minimum $2.5-\mathrm{mm}$ basilic vain diameter and absence of stenosis in the central drainage vein were imposed for BBAVF creation. The basilic vein and brachial artery were evaluated making an approximately $3-\mathrm{cm}$ incision to the medial aspect of the antecubital fossa. In cases with suitable vascular structures, the basic vein was accessed through an incision from the proximal aspect of the elbow in the medial arm to the axillary region and was transected distally as far as possible. The basilic vein was, then, released by ligating the side branches with $3 / 0$ silk sutures. Using this, care was taken to protect the median antebrachial cutaneous nerve from injury. In patients undergoing BVS, following dilation with warm heparinized saline solution, the basilic vein was transported over the deep fascia. Following heparinization, the basilic vein was anastomosed to the brachial artery using an end-to-side technique with $7 / 0$ polypropylene sutures. The basilic vein was brought closer to the underlying deep fascia with individual 2/0 Vicryl sutures (Figure 1). In patients undergoing BVT, a curving subcutaneous tunnel was created in the anterior part of the incision line on the arm with the help of a dilator. The dilated basilic vein was subsequently brought to the distal incision

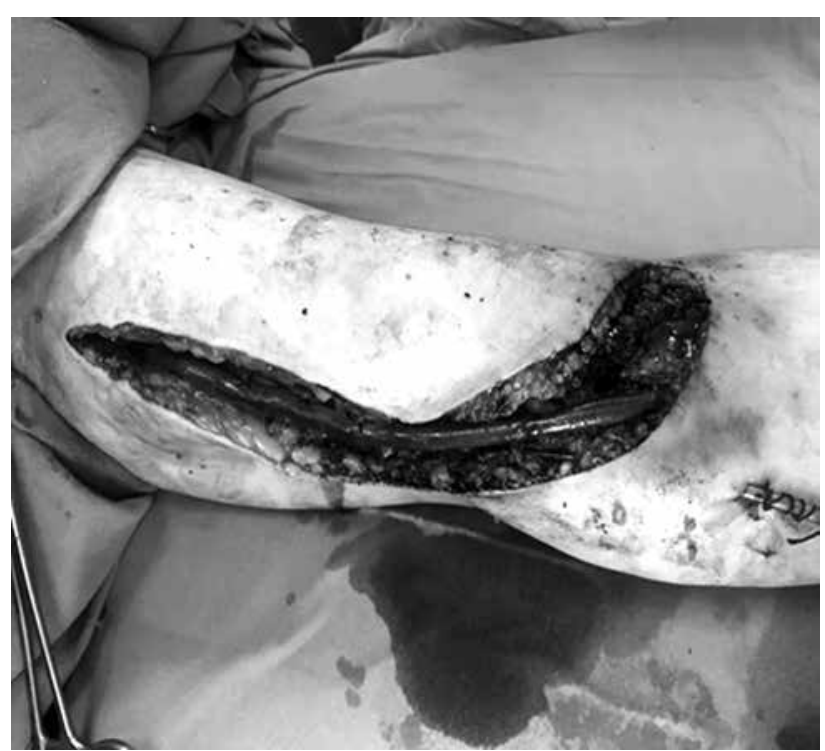

Figure 1. Intraoperative image of basilic vein superficialization in a patient. 
Table 1. Demographic characteristics and preoperative diagnosis of patients

\begin{tabular}{|c|c|c|c|c|c|c|c|}
\hline & \multicolumn{3}{|c|}{ BVS Group (n=42) } & \multicolumn{3}{|c|}{ BVT Group (n=29) } & \multirow[b]{2}{*}{$p$} \\
\hline & $\mathrm{n}$ & $\%$ & Mean \pm SD & $\mathrm{n}$ & $\%$ & Mean \pm SD & \\
\hline Age (year) & & & $57.6 \pm 9.5$ & & & $55.6 \pm 11.5$ & $0.40^{*}$ \\
\hline \multicolumn{8}{|l|}{ Gender } \\
\hline Male & 18 & 43 & & 14 & 48 & & $0.83 * *$ \\
\hline Hypertension & 24 & 57 & & 13 & 45 & & $0.43^{* *}$ \\
\hline Diabetes mellitus & 15 & 36 & & 13 & 45 & & $0.59 * *$ \\
\hline Coronary artery disease & 4 & 10 & & 3 & 10 & & $1.00 * *$ \\
\hline Peripheral artery disease & 12 & 29 & & 7 & 24 & & $0.88^{* *}$ \\
\hline
\end{tabular}

line through this tunnel. Following heparinization, the basilic vein was anastomosed to the brachial artery using an end-to-side technique with $7 / 0$ polypropylene sutures. Following bleeding control, a minivac drain (Bıçakçılar, Istanbul, Turkey) was inserted into the surgical field in all patients. The subcutaneous tissue was subsequently closed with separate $2 / 0$ Vicryl sutures. Postoperative single-dose cefazolin sodium $1 \mathrm{~g}$ was intravenously administered. All patients were discharged one day after surgery.

\section{Statistical analysis}

Statistical analysis was performed using the IBM SPSS version 22.0 software (IBM Corp., Armonk, NY, USA). Continuous variables were expressed in mean \pm standard deviation $(\mathrm{SD})$ and categorical variables were expressed in number and frequency. Since age data were normally distributed in the BVS and BVT groups, comparisons were performed using the t-test. The chi-square test was used, irrespective of normal distribution, to determine whether any significant differences existed between the BVS and BVT groups in terms of preoperative additional diagnoses, primary patency rates, and postoperative complication rates. A $p$ value of 0.05 was considered statistically significant.

\begin{tabular}{|c|c|c|c|c|c|}
\hline & \multicolumn{2}{|c|}{ BVS Group (n=42) } & \multicolumn{2}{|c|}{ BVT Group (n=29) } & \multirow[b]{2}{*}{$p$} \\
\hline & $\mathrm{n}$ & $\%$ & $\mathrm{n}$ & $\%$ & \\
\hline Primary patency & 36 & 86 & 23 & 79 & $0.53^{*}$ \\
\hline Secondary patency & 38 & 90 & 26 & 90 & $1.00^{*}$ \\
\hline Bleeding & 8 & 19 & 5 & 17 & $1.00^{*}$ \\
\hline Thrombosis & 7 & 17 & 4 & 14 & $1.00^{*}$ \\
\hline Edema & 6 & 14 & 5 & 17 & $0.74^{*}$ \\
\hline Aneurysm & 1 & 2 & 1 & 3 & $1.00^{*}$ \\
\hline Infection & 2 & 5 & 2 & 7 & $1.00^{*}$ \\
\hline
\end{tabular}

BVS: Basilic vein superficialization; BVT: Basilic vein transposition; * Chi-square.

\section{RESULTS}

Of 71 patients, 54 (76\%) had a previous history of AVF creation. Seven of these patients received dialysis with a permanent indwelling catheter. The BVS group consisted of 42 patients (18 males, 24 females; mean age 57.6 years, range, 35 to 73 years), and the BVT group of 29 patients (14 males, 15 females; mean age 55.5 years, range, 31 to 76 years). The mean follow-up was 18.8 (range, 6 to 48 months) months. There was no statistically significant difference between the groups in terms of demographic data or preoperative additional diagnoses (Table 1). Bleeding or hematoma developed in 13 patients in the early postoperative period, four of whom requires a surgical intervention. Extremity edema corrected with elevation occurred in 11 patients, and wound site infection treated with antibiotherapy developed in four. Fistula thrombosis in 11 patients during follow-up was treated surgically in six of them and using an interventional method in five. The patency was achieved in five of these patients, while fistula loss occurred in six patients. Primary and secondary patency rates were $86 \%$ and $90 \%$, respectively in the superficialization group and $76 \%$ and $90 \%$, respectively in the transposition group. There was no statistically significant difference in the patency rates or postoperative complications between the groups (Table 2).

\section{DISCUSSION}

Autogenous AVFs using superficial forearm veins have been recommended as the method of choice for vascular access in hemodialysis patients. ${ }^{[1,2,6,9]}$ Considering the importance of autogenous graft use, procedures involving the basilic vein are particularly important in patients in whom there is no possibility of creating an AVF with the cephalic vein and with continuing dialysis requirements. ${ }^{[9,10]}$ After entering the market for use in hemodialysis access, synthetic grafts became popular due to their ease of 
application, a high flow speed, and the absence of any maturation waiting period for dialysis cannulation. The use rates for secondary AVF establishment reached as high as 70 to $80 \% \cdot{ }^{[1,9]}$ Resulting increased complication rates and costs revealed the importance of autogenous grafts, and the recommendation that autogenous grafts should be preferred over synthetic grafts appeared in the guidelines. ${ }^{[1,2,6]}$ Studies have reported much higher patency rates in BBAVFs, while rates of complications such as thrombus and infection are higher in synthetic grafts. ${ }^{[1-3]}$ Infection rates as high as 19\% have been reported with synthetic grafts, and the grafts frequently have to be removed. Meanwhile, synthetic grafts have been linked to greater steal syndrome, aneurysm development, and high-output heart failure. ${ }^{[1]}$ Clear evidence regarding the favorability of BBAVF among secondary AVF options, then, raised the question of which BBAVF procedures is superior. In the present study, we compared the most frequently applied BBAVF techniques among themselves and concluded that there was no significant difference between the BVS and BVT techniques in terms of patency and complications rates.

The natural position of the basilic vein in the deep, medial part of the arm protects it from vascular intervention damage. Its high flow rate and smooth fistula tract make basilic vein AVF creation a viable option. ${ }^{[1-3]}$ The BBABF involves a longer and more complex surgical procedure than other AVF procedures. ${ }^{[3]}$ There is still no consensus regarding whether BBAVF creation should be singleor two-stage, and the decision is mainly left to the surgeon. The advantages of the single-stage procedure are that the patient is taken for surgery only once, and that more rapid functional patency is achieved. With its shorter catheter use time, it also involves a lower risk of complications such as catheter-associated infection and central venous stenosis. The two-stage procedure possesses the advantage that the basilic vein, which is arterialized and, thus, less susceptible to injury, can be easily mobilized. ${ }^{[1,2]}$ Literature data are limited on this subject, although one prospective, randomized study of 40 patients reported primary patency rates of $60 \%$ for the single-stage procedure and $90 \%$ for the two-stage procedure. ${ }^{[11]}$ In contrast, another meta-analysis concluded that the two-stage approach is more popular; however, that there was no statistically significant difference between the single- and two-stage procedures to make it possible to recommend one over the other. ${ }^{[12]}$ In our clinic, we routinely perform $\mathrm{BBAVF}$ procedures in a single stage to achieve more rapid functional patency and owing to low catheter-related complication rates.

Physical examination alone is considered sufficient for patients scheduled for AVF creation using the cephalic vein in our clinic, although imaging techniques are used in case of suspected arterial or venous stenosis. However, since physical examination is not sufficient due to the deep location of the basilic vein, patients scheduled for BBAVF creation are routinely evaluated with Doppler USG and with venography, when necessary, for preoperative assessment and postoperative follow-up. We believe that this approach makes a significant positive contribution to the primary and secondary patency rates of our patients in both groups. In a prospective study involving 59 BVTs, Karakayali et al. ${ }^{[13]}$ reported a one-year secondary patency rate of $92 \%$. This is superior to the patency rates in several studies. The aforementioned authors attributed this positive outcome to the routine use of vascular imaging techniques for preoperative evaluation and postoperative follow-up.

The number of studies comparing BBAVF procedures among themselves in terms of patency rates and complications is extremely limited. ${ }^{[14]}$ In a retrospective study, Hossny ${ }^{[15]}$ evaluated 20 patients undergoing single-stage superficialization, 20 undergoing two-stage superficialization, and 30 undergoing single-stage transposition. Although no significant difference was observed in the patency rates among the groups, overall complication rates were significantly higher in patients undergoing superficialization than in those undergoing transposition (71.4 vs. $28.6 \%$, respectively; $\mathrm{p}<0.001$ ). In addition, lower satisfaction rates among dialysis personnel and greater patient complaints were reported in an elevation group for several reasons such as difficult cannulation. In their meta-analysis, Dukkipati et al. ${ }^{[2]}$ described hematoma as the most important complication affecting fistula failure, and reported that hematoma was present in $63.7 \%$ of thrombosed fistulas. Hematoma was more common in the BVS group than in the BVT group in that study (26.3 vs. $3.6 \%$, respectively). In the present study, although the complications of postoperative bleeding and fistula thrombosis were more common in the BVS group compared to the BVT group, the difference was not statistically significant. In addition, upper extremity edema was seen in 11 patients in the early postoperative period, and incision line infection in four. All the upper extremity edemas resolved entirely with elevation, and all cutaneous infections resolved entirely with antibiotherapy (Table 2). 
Basilic vein stenosis that may occur following BBAVF procedures is associated with complications such as bleeding, edema, fistula thrombosis, and aneurysm formation. Stenosis often occurs in the swing segment of the proximal basilic vein in patients undergoing BVT. On the other hand, stenosis of the basilic vein in patients not undergoing transposition and with only superficialization is rare. However, the basilic vein immediately beneath the incision line has to be cannulated from the scar tissue, and this is associated with the development of additional complications. Interventional or surgical procedures are needed to treat stenotic lesions in the basilic vein. ${ }^{[14,16,17]}$ In the present study, fistula thrombosis developing due to basilic vein stenosis in 11 patients during follow-up, seven undergoing BVS and four undergoing BVT, was treated surgically in six and with interventional methods in five patients. Patency was achieved in five of these patients, while fistula loss occurred in six. A previous study described a novel approach referred to as basilic elevation transposition (BET) to avert stenosis caused by transposition. Using this approach, the superficialized basilic vein is inserted into a pocket beneath the skin in front of the cutaneous incision. In this way, in addition to reducing the risk of basilic vein kinking, it was also reported to be inserted into healthy subcutaneous tissue. In that study, the primary patency rate was significantly higher in patients undergoing $\mathrm{BET}$ compared to those undergoing BVT, while the number of percutaneous angioplasties required for the basilic vein was significantly lower in the BET group. The authors concluded that high patency rates could be achieved with both BVT and BET, and that BBAVF outcomes could be improved with the described technique by reducing angulation and torsion in the basilic vein. ${ }^{[14]}$

Nonetheless, there are some limitations to the present study, including its retrospective and nonrandomized nature, relatively small sample size and the fact that the sample sizes were unequal, and the absence of data for longer-term follow-up periods. Further prospective, large-scale studies comparing the BVS and BVT procedures are needed to elicit clinically useful results.

In conclusion, both the BVS and BVT procedures can be safely applied in patients in whom arteriovenous fistula cannot be created using the cephalic vein, owing to their low complication and high patency rates. The use of ultrasonography at both preoperative evaluation and postoperative follow-up can make a significant contribution to the improved patency rates. Our results suggest that there is no significant difference between the two methods in terms of complication and patency rates, and that neither is superior to the other. We recommend further, larger scale, prospective studies to confirm these findings.

\section{Declaration of conflicting interests}

The authors declared no conflicts of interest with respect to the authorship and/or publication of this article.

\section{Funding}

The authors received no financial support for the research and/or authorship of this article.

\section{REFERENCES}

1. Dix FP, Khan Y, Al-Khaffaf H. The brachial arterybasilic vein arterio-venous fistula in vascular access for haemodialysis--a review paper. Eur J Vasc Endovasc Surg 2006;31:70-9.

2. Dukkipati R, de Virgilio C, Reynolds T, Dhamija R. Outcomes of brachial artery-basilic vein fistula. Semin Dial 2011;24:220-30.

3. Hastaoğlu İO, Erdem CÇ, Bilgen F. Transposition of dorsally lying forearm basilic vein for distal hemodialysis vascular access in the upper extremity: Surgical technique and mid-term results. Turk J Vasc Surg 2012;21:1-5.

4. Yüksel V, Halıcı Ü, Hüseyin S, Güçlü O, Canbaz S, Ege $\mathrm{T}$, et al. Basilic vein superficialization for hemodialysis vascular access. Turk Gogus Kalp Dama 2013;21:950-4.

5. Uzun HA, Çiçek ÖF, Seren M. Transposition of basilic vein in forearm for arteriovenous fistula creation: Our midterm results. Turk Gogus Kalp Dama 2019;27:508-11.

6. Schmidli J, Widmer MK, Basile C, de Donato G, Gallieni M, Gibbons CP, et al. Editor's Choice - Vascular Access: 2018 Clinical Practice Guidelines of the European Society for Vascular Surgery (ESVS). Eur J Vasc Endovasc Surg 2018;55:757-818.

7. Dagher F, Gelber R, Ramos E, Sadler J. The use of basilic vein and brachial artery as an A-V fistula for long term hemodialysis. J Surg Res 1976;20:373-6.

8. Francis DM, Lu Y, Robertson AJ, Millar RJ, Amy J. Two-stage brachiobasilic arteriovenous fistula for chronic haemodialysis access. ANZ J Surg 2007;77:150-5.

9. İşcan Ş, Gürsu Ö, Etli M. Is basilic vein transposition now the first choice ? Short term results of a minimally invasive technique. Turk J Vasc Surg 2013;22:280-4.

10. El Sayed HF, Mendoza B, Meier GH, LeSar CJ, DeMasi RJ, Glickman MH, et al. Utility of basilic vein transposition for dialysis access. Vascular 2005;13:268-74.

11. E1 Mallah S. Staged basilic vein transposition for dialysis angioaccess. Int Angiol 1998;17:65-8.

12. Bashar K, Healy DA, Elsheikh S, Browne LD, Walsh MT, Clarke-Moloney M, et al. One-stage vs. two-stage brachio-basilic arteriovenous fistula for dialysis access: a systematic review and a meta-analysis. PLoS One 2015;10:e0120154. 
13. Karakayali FY, Sevmis S, Basaran C, Yabanoglu H, Arat $\mathrm{Z}$, Boyvat $\mathrm{F}$, et al. Relationship of preoperative venous and arterial imaging findings to outcomes of brachiobasilic transposition fistulae for hemodialysis: a prospective clinical study. Eur J Vasc Endovasc Surg 2008;35:208-13.

14. Wang S, Wang MS, Jennings WC. Basilic elevation transposition may improve the clinical outcomes for superficialization of basilic arteriovenous fistula veins. J Vasc Surg 2017;65:1104-12.

15. Hossny A. Brachiobasilic arteriovenous fistula: different surgical techniques and their effects on fistula patency and dialysis-related complications. J Vasc Surg 2003;37:821-6.

16. Bronder CM, Cull DL, Kuper SG, Carsten CG, Kalbaugh $\mathrm{CA}$, Cass A, et al. Fistula elevation procedure: experience with 295 consecutive cases during a 7-year period. J Am Coll Surg 2008;206:1076-81.

17. Kakkos SK, Kouri AK, Tsolakis IA, Haddad GK, Lampropoulos GC, Karnabatidis D. Surgical and endovascular revision of brachio-basilic vein fistula. J Vasc Access. 2016;17:S6-11. 